PACS 05.60, 67.60

\title{
HEAT TRANSFER AND DIFFUSION IN MULTICOMPONENT CLASSICAL AND QUANTUM SYSTEMS
}

\author{
I.N.ADAMENKO, K.E.NEMCHENKO \\ Department of Physics and Technology, Kharkiv State University \\ UA-310077 Kharkiv, Ukraine
}

Received September 12, 1995; revised May 11, 1996

\begin{abstract}
Diffusion matrix of three-component mixture of particles with arbitrary dispersion laws and statistics is found. The coefficients of mass and spin diffusion, thermal diffusion, and thermal conductivity of superfluid helium isotopes mixtures are derived from the obtained general relations. Results of calculations are compared with experimental data.
\end{abstract}

\section{Diffusion coefficients in classical gas mixture}

We consider a stationary nonequilibrium state of three - component classical gas mixtures in which the number densities $n_{i}$ of $\mathrm{i}$-th component $(\mathrm{i}=1,2,3)$ are functions of coordinates. In a three-component mixture the coefficients of diffusion form matrix $D_{i k}$ which determines the dependence of dissipative flow velocities $\vec{U}_{j}$ of each components $(\mathrm{j}=1,2,3)$ on gradients of $n_{k}$ :

$$
\vec{U}_{j}=-\sum_{k=1}^{3} D_{j k} \frac{\nabla n_{k}}{n}
$$

where $n=n_{1}+n_{2}+n_{3}$ is the total number density of the particles in the mixture. To calculate $D_{j k}$ the system of linearized kinetic equations has been solved by using projective operators technique developed in [1]. The final results for $D_{j k}$ are

$$
D_{j k}=\frac{n T}{\rho} t_{j k},
$$

where $\rho=\sum_{l=1}^{3} \rho_{l}=\sum_{l=1}^{3} m_{l} n_{l}$ is the mass density of the mixture;

$$
\begin{gathered}
t_{j j}=\Delta_{k l}^{-1}\left[\frac{\rho_{j}}{\rho} t_{l}^{-1}+\frac{\rho_{l}}{\rho} t_{k}^{-1}\right], \\
t_{j k}=-\Delta_{k l}^{-1}\left[\frac{\rho_{j}}{\rho}\left(t_{l}^{-1}-\tau_{j l}^{-1}\right)-\frac{\rho_{l}}{\rho} \tau_{k j}^{-1}\right], \\
t_{l}=\tau_{l j}^{-1}+\tau_{l k}^{-1}+\tau_{k l}^{-1} ; \quad \Delta_{k l}=\tau_{k j}^{-1} \tau_{l k}^{-1}+\tau_{l j}^{-1} \tau_{k l}^{-1}+\tau_{k j}^{-1} \tau_{l j}^{-1} .
\end{gathered}
$$


Here $j \neq l \neq k ; j, k, l \in\{1,2,3\}$;

$$
\tau_{k l}^{-1}=\left\langle\vec{p}_{k} \mid \vec{p}_{k}\right\rangle^{-1}\left\langle\vec{p}_{k}\left|I_{k l}\right| \vec{p}_{k}\right\rangle
$$

are the collision rates of $k$-th particle with $l-t h$ particle, $\vec{p}_{k}$ is the momentum. In (6) the following scalar product was introduced

$$
\langle\psi \mid \chi\rangle=-\int \psi^{*}\left(\vec{p}_{k}\right) \chi\left(\vec{p}_{k}\right) f_{k}^{\prime} d \Gamma_{k},
$$

where $f^{\prime}{ }_{K}$ is the derivative of local-equilibrium distribution function $f_{k}$ with respect to energy, $d \Gamma_{\pi}$ is a phase volume element. The linear collision operator $I_{k l}$ is defined by corresponding collision integrals. For usual binary collision integral with the transition probability density $w\left(\vec{p}_{k}, \vec{p}_{l} ; \vec{p}_{k}{ }^{\prime}, \vec{p}_{l}{ }^{\prime}\right)$ we have

$$
I_{k l}\left|\vec{p}_{k}\right\rangle=\left|\int w f_{l}^{\prime}\left(\vec{p}_{k}-\vec{p}_{k}{ }^{\prime}\right) d \Gamma_{l} d \Gamma_{k} d \Gamma_{k}^{\prime}\right\rangle .
$$

From (6) and the momentum conservation law we have

$$
\tau_{k l}^{-1}=\tau_{l k}^{-1} \frac{\rho_{l}}{\rho_{k}} .
$$

Expression (2) can be obtained from the relations presented in [2], where the results are expressed as a system of equations with integral brakets, which, in fact, are analogous to matrix elements (6). However, expressions (2) for $D_{j} k$ written by using the relaxation rates $\tau_{k l}^{-1}$ are more suitable for discussion, allow to explain physical meaning of all the results obtained, to compare them with those for the gas of quasiparticles, and to consider various limiting cases.

\section{Diffusion and heat transfer in the superfluid mixture of helium isotopes}

There may exist mass and spin diffusion flows of the light helium isotope in superfluid ${ }^{3} \mathrm{He}-{ }^{4} \mathrm{He}$ solutions. The kinetic properties of such a mixture [5] are completely defined by a three-component gas of quasiparticles. They are phonons, rotons (thermal exitations) and impuritons (quasiparticles of ${ }^{3} \mathrm{He}$ ). To calculate the coefficients of diffusion and diffusion thermal conductivity of a superfluid mixture one has to solve, as in the case of three-component classical gas, the system of three kinetic equations for distribution functions of phonons $f_{p h}$, rotons $f_{r}$ and impuritons $f_{i}$. As the result the velocities of dissipative flows $\vec{U}_{j}$ may be written in the way which can be used both for the mixture of quasiparticles of quantum ${ }^{3} \mathrm{He}-{ }^{4} \mathrm{He}$ solutions and that of classical gases.

$$
\vec{U}_{j}=-\sum_{k=1}^{3} \frac{1}{\rho} t_{j k} \frac{\nabla P_{k}}{n},
$$

where $P_{k}$ is the partial pressure. For classical gases the equality $\nabla P_{k}=$ $T \nabla n_{k}$ gives relation (1). In the case of quasiparticles one should have in mind that index 1 corresponds to phonons, 2 to rotons, and 3 to impuritons. For the partial pressure we have

$$
\nabla P_{p h}=S_{p h} \nabla T, \nabla P_{r}=S_{r} \nabla T, \nabla P_{i}=\nabla\left(n_{i} T\right),
$$


where $S_{p h}$ and $S_{r}$ are the entropies of phonons and rotons respectively. Substituting (11) in (10) with $j=1 \rightarrow i$ and taking into account the definition [3] or coefficients of mass diffusion $D_{m}$ and thermal diffusion ratio $k_{T}$ we obtain

$$
\begin{gathered}
D_{m}=\frac{T}{m}\left(\frac{\rho_{t}}{\rho_{n}}\right)^{2} \tau_{i t}, \\
k_{T}=c \frac{m}{T}\left[\frac{n_{i} T}{\rho_{i}}-\frac{S_{t} T}{\rho_{t}}+\frac{\rho_{p h} \rho_{r}}{\rho_{i} \rho_{t}}\left(\frac{S_{p h} T}{\rho_{p h}}-\frac{S_{r} T}{\rho_{r}}\right) \tau_{\kappa}\left(\tau_{p h i}^{-1}-\tau_{r i}^{-1}\right)\right],
\end{gathered}
$$

where $\rho_{n}=\rho_{t}+\rho_{i}=\rho_{p h}+\rho_{r}+\rho_{i}$ is the normal density of the mixture $\left(\rho_{i}=m_{i} n_{i}\right) ; c=\rho_{i} /\left(\rho_{i}+m_{4} n_{4}\right)$ is the mass concentration of impurities; $S_{t}=S_{p h}+S_{r}$ is the entropy of thermal excitations

$$
\begin{gathered}
\tau_{i t}=\frac{\rho_{i}}{\rho_{t}} \Delta^{-1}\left[\frac{\rho_{p h}}{\rho_{t}} t_{r}^{-1}+\frac{\rho_{r}}{\rho_{t}} t_{p h}^{-1}\right]=\frac{\rho_{i}}{\rho_{t}} \Delta^{-1} \tau_{\kappa}, \\
\Delta=\tau_{p h i}^{-1} \tau_{r i}^{-1}+\tau_{p h r}^{-1} \tau_{r i}^{-1}+\tau_{r p h}^{-1} \tau_{p h i}^{-1}, \\
t_{r}^{-1}=\tau_{r i}^{-1}+\tau_{r p h}^{-1}+\tau_{p h r}^{-1}, \\
t_{p h}{ }^{-1}=\tau_{p h i}^{-1}+\tau_{p h r}^{-1}+\tau_{r p h}^{-1} .
\end{gathered}
$$

The thermal diffusion ratio turnes out to be dependent on the interactions rates between quasiparticles. This dependence is due to the difference in the rates of phonons and rotons interactions with impuritons.

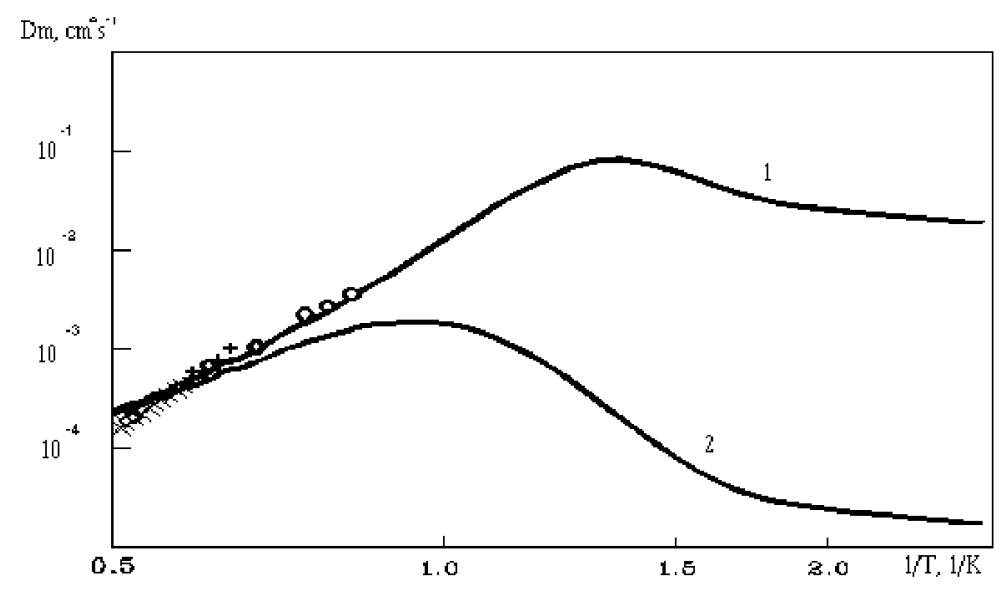

Figure 1. Temperature dependence of mass diffusion coefficient, calculated from equation (12) at different concentrations: 1) $x=10^{-3}$;2) $x=10^{-2}$ and experimental data from [4] at molar concentrations $1.39 \cdot 10^{-4}(\mathrm{o})$ and $1.32 \cdot 10^{-3}(\diamond)$ and from $[5]$ at $9.4 \cdot 10^{-4}(\times)$ and $1.4 \cdot 10^{-3}(+)$.

Figure 1 shows the mass diffusion coefficient as a function of the reciprocal temperature according to equation (12). Existing experimental data are plotted in the same figure. The presence of a maximum on the curve is due to the bell - curve dependence contained in the function $\rho_{t} \rho_{n}^{-2}$, which attains the maximum for $\rho_{t}=\rho_{i}$, and to the temperature dependence of the 
factor $\rho_{t} \tau_{i t}$. When analysing this dependences it is useful to consider the product of time and corresponding density. This product depends weakly (and in several cases not at all) on the densities of the mixture components.

Using expression (10) one can find the contribution of diffusion between thermal excitations to thermal conductivity. According to [1] the coefficient of thermal conductivity of the superfluid ${ }^{3} \mathrm{He}-{ }^{4} \mathrm{He}$ mixture may be written as

$$
\kappa=\kappa_{i}+\kappa_{p h}+\kappa_{r}+\kappa_{p h, r, i}
$$

where $\kappa_{i}, \kappa_{p h}$, and $\kappa_{r}$ are the so-called partial coefficients of thermal conductivity which determine the additive contribution of impuritons, phonons, and rotons respectively, and $\kappa_{p h, r, i}$ is the so-called [1] nonadditive part of thermal conductivity. When the phonon dispersion law is linear the coefficient $\kappa_{p h}=0$.

The diffusion part of thermal conductivity $\kappa_{D}$ may be determined as a coefficient between the heat flow $\vec{q}_{t}$ of thermal excitations

$$
\vec{q}_{t}=S_{p h} T \vec{u}_{p h}+S_{r} T \vec{u}_{r}
$$

and the temperature gradient. This coefficient $\kappa_{D}$ generally [3] is defined, when the impuriton mass flow $\vec{u}_{i}=0$ and

$$
\left.\mathbf{q}_{t}\right|_{\mathbf{U}_{i}=0}=-\kappa_{D} \nabla T \text {. }
$$

This relation, together with (15), gives

$$
\kappa_{D}=\frac{1}{T}\left\{\frac{S_{p h} T}{\rho_{p h}}-\frac{S_{r} T}{\rho_{r}}\right\}^{2} \frac{\rho_{p h} \rho_{r}}{\rho} \tau_{D} .
$$

Comparison of (18) with the result (15) from [1] calculated starting from the definition of coefficients of thermal conductivity in the ${ }^{3} \mathrm{He}-{ }^{4} \mathrm{He}$ superfluid mixture gives $\kappa_{D}=\kappa_{p h, r, i}$. So the nonadditive part of thermal conductivity is due to diffusion between thermal excitations.

Note the symmetry of formula (18) with respect to exchange phonons and rotons. In pure ${ }^{4} \mathrm{He}$ the expression for $\kappa_{p h, r, i}$ can be written in a nonsymmetric way

$$
\kappa_{D n_{i}=0}=\frac{1}{T} \rho_{p h} v_{p h}^{4}\left(1-\frac{T S_{t}}{\rho_{t} v_{p h}^{2}}\right)^{2} \tau_{\kappa}
$$

presented in [3]. This nonsymmetric formula gave the reason to call (19) the "phonon" thermal conductivity. The symmetry of (19) proves that this name is especially conventional even in pure ${ }^{4} \mathrm{He}$, not saying about the mixtures when $\kappa_{D}$ depends on $\tau_{r i}$. So, considering the diffusion in the gas of thermal excitations one can obtain the "phonon" part of thermal conductivity.

Estimations show that in wide temperature and concentration range $\kappa_{D}$ turnes out to be much greater than the partial thermal conductivity $\kappa_{i}$ and $\kappa_{r}$. In this case thermal conductivity of the mixture is completely determined by diffusion flow of phonons and rotons. According to (18) $\kappa_{D}$ is affected by the difference between dispersion laws of thermal excitations. The same mechanism is supposed to take place in other multicomponent gases of quasiparticles. 


\section{Spin diffusion in the superfluid mixture of helium iso- topes}

From the relation (10) one can easily find the coefficient of spin diffusion at low temperatures, where rotons can be neglected. It is enough to suppose $j=1$ and 1 corresponds to $\uparrow$ (spin up), 2 to $\downarrow$, and 3 to subscript $p h$. The term including the roton-impuriton relaxation rate is obtained from the solution of the same problem for a four-component gas of quasiparticles:

$$
D_{S}=\frac{T}{m}\left(\tau_{i i}^{-1}+\tau_{i p h}^{-1}+\tau_{i r}^{-1}\right)^{-1},
$$

where $\tau_{i i}$ is the impuriton relaxarion rate.

As far as we know the spin diffusion coefficient of superfluid mixtures of ${ }^{3} \mathrm{He}-{ }^{4} \mathrm{He}$ has not been yet calculated with taking account of thermal excitations. It was presented in [4] by empirical relation:

$$
D_{S}^{-1}=D^{-1}+D_{i i}^{-1},
$$

where D was considered to be the coefficient of mass diffusion, and $D_{i i}$ is defined by interactions of impuritons.

The result (20) can be written in the form (21) to give

$$
\begin{gathered}
D=\frac{T}{M}\left(\tau_{i p h}^{-1}+\tau_{i r}^{-1}\right)^{-1}, \\
D_{i i}=\frac{T}{m} \tau_{i i} .
\end{gathered}
$$

The expression (22) essentially differs from the coefficient of mass diffusion (12). The presence of the factor $\rho_{t}^{2} \rho_{n}^{-2}$ in $D_{m}$ is the main difference between (22) and (12) in respect with temperature dependence. This dependence of $D_{m}$, unlike $D$, has bell-like shape (see figure 1). Furthermore the times in these coefficients are different. The coefficient $D$ includes usual kinetic combination

$$
\tau_{S}=\left(\tau_{i p h}^{-1}+\tau_{i r}^{-1}\right)^{-1}
$$

of relaxation rates with phonons and rotons but $D$ includes time (14). This is the relaxation time in three-component gas of quasiparticles. The combination $\tau_{i t}$ depends not only on the interactions of impuritons with thermal excitations but on the phonon-roton relaxation, as well. In general, the difference between relaxation-time equations (24) and (14) becomes clear when the latter is written in the form

$$
\tau_{i t}=\left[\tau_{i p h}^{-1}+\tau_{i r}^{-1}-\frac{\rho_{p h} \rho_{r}}{\rho_{i} \rho_{n}} \tau_{\kappa}\left(\tau_{p h i}^{-1}-\tau_{r i}^{-1}\right)^{2}\right]^{-1} .
$$

Thus, the time $\tau_{S}$ is always less than $\tau_{i t}$ to the extent of the difference between the collision rates of impuritons with rotons and phonons.

According to equation (20), the spin diffusion coefficient is determined mainly by the time $\tau_{i i}$ at sufficiently low temperatures. To calculate this time, one has to know the impuriton-impuriton interaction potential, for which a generally accepted expressions do not exist at present. For this 
reason we determine $\tau_{i i}$ from the observed values [4] of the spin diffusion in the low-temperature range to have:

$$
\tau_{i i}=5 \cdot 10^{-12} T^{-2} x^{-1} .
$$

The observed and calculated from (20) values of spin diffusion coefficient are shown in figure 2 for various concentrations. Curves $4^{\prime}$ and $5^{\prime}$ in the same figure are plotted according to equation (21), where $D$ is assumed to be the mass diffusion coefficient. The comparison of equations (12) and (22) shows that $D_{m}$ coincides with $D$ only in the case $x \rightarrow 0$. This result confirms the processing of the experiments for $T>0.8 K$, when $D$ can still be determined with acceptable accuracy from data on $D_{s}$. The agreement of curves $1,2,3,4$, and 5 with the experimental data and deviation of curves $4^{\prime}$ and $5^{\prime}$ are strong evidence in favor of the reliability of the results obtained in this study.

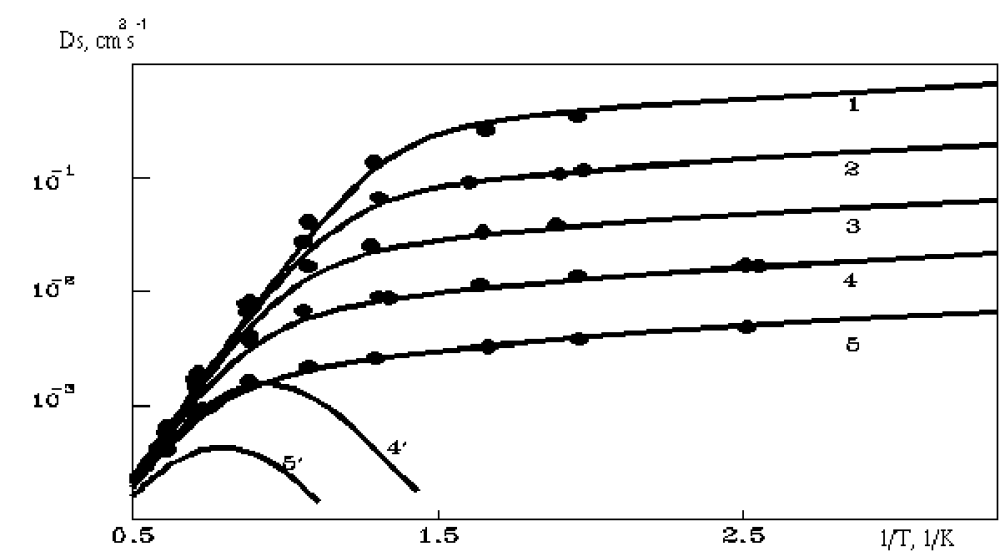

Figure 2. Temperature dependence of spin diffusion coefficient, experimental data from [4] $(\bullet)$ for various concentrations: 1) $3 \cdot 10^{-4}$; 2$) 1.02 \cdot 10^{-3}$; 3$) 3.1 \cdot 10^{-3}$; 4) $9.2 \cdot 10^{-3}$; 5) $3 \cdot 10^{-2}$, calculated from equation (20) at the same concentrations (curves 1-5, respectively), and calculated from equation (21) with $D$ as the mass diffusion coefficient (12) (curves $4^{\prime}$ and $5^{\prime}$ ) from equation (20).

\section{References}

[1] Adamenko I.N., Nemchenko K.E., Tsyganok V.I. Dissipative processes and second sound in ${ }^{3} \mathrm{He}-{ }^{4} \mathrm{He}$ superfluid mixtures. // J. of Low Temp. Phys., 1990, vol. 81 , No 5/6, p. 233-262.

[2] Ferziger J.H., Kaper H.G. Mathematical theory of transport in gases. Elsevier, North-Holland, New-York, 1972.

[3] Khalatnikov I.M. Introduction in the theory of superfluidity. Benjamin, NewYork, 1965.

[4] Esel'son B.N., Ivantsov V.G., Koval V.A., Rudavskii E.Ya., Serbin I.A. Properties of solid and liquid helium. Naukova Dumka, Kiev, 1982 (in Russian).

[5] Tuttle J., Zhong F., Meyer H. Relaxation times and mass diffusion in superfluid dilute ${ }^{3} \mathrm{He}-{ }^{4} \mathrm{He}$ mixtures. // J. of Low Temp. Phys., 1991, vol. 82, No 1/2, p. $15-29$. 


\title{
ТЕПЛОВИЙ ПЕРЕНОС ТА ДИФУЗІЯ У БАГАТОКОМПОНЕНТНИХ КЛАСИЧНИХ ТА КВАНТОВИХ СИСТЕМАХ
}

\author{
I.М.Адаменко, К.Е.Немченко
}

Обчислена дифузійна матриця трикомпонентної суміші частинок 3 довільним законом дисперсії та статистикою. Із отриманих загальних виразів знайдені коефіцієнти спінової, масової дифузії, термодифузії та теплопровідності надплинних розчинів ізотопів гелію. Результати обчислень порівнюються з експериментальними даними. 